\title{
Efficient Key Authentication Service for Secure End-to-end Communications
}

\author{
Mohammad Etemad \\ Alptekin Küpçü \\ Crypto Group, Koç University, Istanbul, Turkey \\ \{metemad, akupcu\}@ku.edu.tr
}

\begin{abstract}
After four decades of public key cryptography, both the industry and academia seek better solutions for the public key infrastructure. A recent proposal, the certificate transparency concept, tries to enable untrusted servers act as public key servers, such that any key owner can verify that her key is kept properly at those servers. Unfortunately, due to high computation and communication requirements, existing certificate transparency proposals fail to address the problem as a whole.

We propose a new efficient key authentication service (KAS). It uses server-side gossiping as the source of trust, and assumes servers are not all colluding. KAS stores all keys of each user in a separate hash chain, and always shares the last ring of the chain among the servers, ensuring the users that all servers provide the same view about them (i.e., no equivocation takes place). Storing users' keys separately reduces the server and client computation and communication dramatically, making our KAS a very efficient way of public key authentication. The KAS handles a key registration/change operation in $O(1)$ time using only $O(1)$ proof size; independent of the number of users. While the previous best proposal, CONIKS, requires the client to download $100 \mathrm{~KB}$ of proof per day, our proposal needs less than $1 \mathrm{~KB}$ of proof per key lifetime, while obtaining the same probabilistic guarantees as CONIKS.
\end{abstract}

Keywords: Certificate transparency, End-to-end encryption, Key authentication.

\section{Introduction}

Many online services, ranging from financial services to health and social services, process sensitive data. Most of the sensitive information is transferred through the network, unencrypted or point-to-point encrypted [9]. The former reveals the data to everyone while the latter reveals the data only to those who perform decryption and re-encryption on the path to the destination. One of the breakthroughs to this regard was the invention of public key cryptography $[2,11]$. It is employed in end-to-end secure email and end-to-end encryption (E2EE). To provide security and prevent misuse, the data should be encrypted by the sender, transmitted encrypted, and decrypted by the receiver.

As the networking technologies progress, more and more useful and interesting applications and services, ranging from financial services to health services to social services, are offered online, and hence, more and more people are incentivized to use those services. Most of these service require sensitive information that is transferred through the network, unencrypted or point-to-point encrypted [9]. The former reveals the data to everyone while the latter reveals the data only to those who perform decryption and re-encryption on the path to the destination. To provide security and prevent misuse, the data should be encrypted by the sender, transmitted encrypted, and decrypted by the receiver.

End-to-end secure email. Email is one of the oldest IT services for fast communication. Most people use free email services offered by different email providers such as Google (Gmail) or Yahoo. Gmail initially launched with HTTPS support and uses an encrypted connection for sending and receiving emails, but the encryption is not end-to-end, i.e., the Gmail server has access to the plaintext of all emails that are not encrypted explicitly by the sender. 
End-to-end encryption (E2EE) is required to solve the above-mentioned problem. Having easy access to the target's key, a party can encrypt a message directly with it. E2EE is being considered as an important building block in many recent schemes. Weber [13] used attribute-based encryption for secure end-to-end one-to-many mobile communication. Snake [1] is proposed as an end-to-end encrypted online social networking scheme, relying on establishment of shared keys between users at the start phase of friendship. E2EE is also used in several messaging service such as Apple iMessage and BlackBerry protected messenger [9]. PGP is an end-to-end secure email method which did not get enough traction due to hardness of use and complicated setup requirements. The main problem with all these schemes is key management.

One caveat of public key cryptography is that it requires the communicating parties to obtain genuine keys of each other. If the communicating parties had a secure physical channel (i.e., they could physically meet), then they could have exchanged their public keys. Unfortunately, in the online world, a trusted party called certificate authority (CA) is required. A CA ensures the parties via issuing unforgeable cryptographic evidences (certificates) that the public key contained in the certificate does indeed belong to the party stated in the certificate. However, a main problem with CAs is that they must be trusted, and sometimes this trust is broken [12]. Moreover, there are other applicability and usage problems such as storage, revocation, and distribution [10]. CAs are also valuable targets for adversaries through which they can attack the whole system. We need a new source of trust that cannot easily be compromised legally or under pressure [5], without being detected and revealed.

Certificate transparency is recently proposed to substitute the existing certificate authorities [7]. There is no single source of trust and all users and servers contribute to provide a web of trust among themselves. Following (efficient) schemes [12,9] added key revocation support, and used it as a general key management scheme for different applications such as end-to-end encrypted email. However, all these schemes impose a heavy computation and communication burden on both clients and servers, preventing its mass application. We propose an efficient key authentication service that performs key registration/change operations optimally (i.e., with $O(1)$ computation and communication).

Our contributions are as follows:

- We give the first security definition of a key authentication service, and prove our construction's security formally.

- Our KAS scheme performs key registration/change operations optimally with $O(1)$ server and client computation and $O(1)$ communication. Hence, we reduce the computation and communication costs of the existing schemes [7, 12,9] for each key registration/change operation by a factor of $\log n$, where $n$ is the number of users.

- The total cost of the server in the previous schemes is $O(n \log n)$, since they compute the proof for all users on each key registration/change [7, 12] or on each epoch [9]. A similar audit operation costs $O(n)$ in our KAS.

- Our scheme is perfectly privacy-preserving, i.e., proof of a key (of a user) does not depend on any other key (of another user), and hence, does not reveal any information about other users.

- For the first time, we consider non-repudiation for both client and server. In our scheme, a malicious client cannot frame an honest server.

\subsection{Related Work}

Laurie et al. [7] proposed certificate transparency for preventing certificate misuse by publishing the issued TLS certificates through publicly-auditable logs. The clients and some special entities (called monitors and auditors) will report any misbehavior through regularly verifying the logs. The logs are append-only Merkle hash trees. The tree has the property that any previous version is a subset of all following versions, through which the consistency of logs can be proven. On receipt of a new certificate, the log server appends it into the existing tree, commits to its new root, and returns both the new root and its commitment. The issuing user verifies the result and if it is correct, spreads it through global gossiping. All later verifications should be made against this new root. 
Later, Laurie and Kasper [6] proposed revocation transparency, similar to certificate transparency, as a transparent mechanism for providing the list of revoked certificates and showing that all clients see the same list of revoked certificates. However, it is not efficient due to its space and time complexity that is linear in the number of revocations.

Ryan [12] proposed enhanced certificate transparency (ECT) that can also be used for secure endto-end email or messaging without requiring a trusted certificate authority. ECT also solves the certificate revocation problem efficiently. There is an append-only Merkle tree as in [7] for handling certificate transparency, but in addition, there is another lexicographically ordered hash tree storing a constant number of revoked keys for each client, reducing the revocation complexity from $O(n)$ to $O(\log n)$, where $n$ is the number of users.

Melara et al. [9] proposed CONIKS as an automated key management system. It consists of a number of key providers storing user-to-key bindings of users. The users can detect equivocations or unexpected key changes made by malicious providers. CONIKS provides the following security properties: nonequivocation, binding consistency, privacy-preserving bindings, and security with human-readable names. The following consistency proofs are also provided: proofs of binding consistency, proofs of absence, and checks for non-equivocation.

Laurie et al. [7] and ECT [12] use client-side gossiping, which requires all clients to gossip with each other. This is very inefficient as it is required on each key change. Moreover, it requires a fully connected graph over all users during the gossiping. If the user are partitioned during gossiping, the server can equivocate since the others has no idea about the change that happened. CONIKS [9] uses serverside gossiping, which is much more efficient than the client-side gossiping, especially assuming a constant number of servers.

A problem with these schemes is the lack of formal security definitions and proofs. Moreover, organizing the keys in a tree data structure ties them altogether, which means that even if only one key changes, all users (except the one whose key has been changed) need to check the resulting new tree to make sure they are not affected. We store the user-to-key bindings separately, thus decreasing provider and client computation while increasing the privacy-preservation level. Another problem is that the non-repudiation property for all parties was not considered previously: one cannot show the origin of any potential inconsistency. A malicious client can try to frame an honest server, and neither party could provide evidence of honest behavior. We also address this issue. A comparison of these schemes is given in Table 1 .

Table 1: A comparison of certificate transparency schemes. NR and PP stand for non-repudiation and privacypreserving, respectively, and ' $X$ ' means not fully supported.

\begin{tabular}{|l|c|c|c|c|c|c|}
\hline Scheme & Update & Audit & Revocation & Gossiping & NR & PP \\
\hline Laurie et al. [7] & $O(\log n)$ & $O(n \log n)$ & $O(n)$ & Client-side & - & - \\
\hline ECT [12] & $O(\log n)$ & $O(n \log n)$ & $O(\log n)$ & Client-side & - & - \\
\hline CONIKS [9] & $O(\log n)$ & $O(n \log n)$ & $O(\log n)$ & Server-side & $\times$ & $\times$ \\
\hline Our KAS & $O(1)$ & $O(n)$ & $O(1)$ & Server-side & + & + \\
\hline
\end{tabular}

On a separate line of work, Wendlandt et al. [14] proposed Perspectives for authenticating a server's public key. It uses a number of (semi-)trusted hosts named network notaries that probe most of network services (e.g., SSH) and keep a history of their public keys over time. On receipt of an unauthenticated public key from a service, the client asks the notaries the history of keys used by that service to help her decide on whether to accept or reject it. Perspectives differs from the certificate transparency as it requires all notaries to be trusted.

\subsection{Model}

There are two parties in our model. The providers are the publicly available servers storing name-to-key bindings of the users. There are multiple providers each running in a different domain. Each provider man- 
ages a distinct set of clients ${ }^{1}$, and shares information about them with other providers by gossiping, through a specific PKI. $\mathcal{P}$ represents the set of all providers. We use the terms provider and server interchangeably. For easier presentation, home provider is the provider a user registers her keys with.

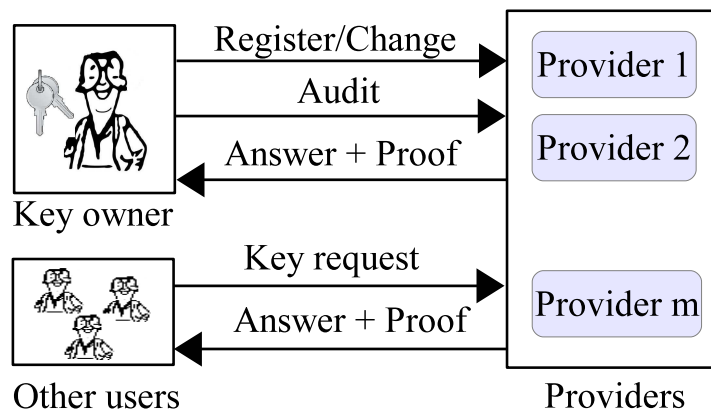

Figure 1: Our model.

The client (or her device on her behalf) registers her (public) key in a provider and can access the keys of other clients through the (same or other) provider(s). We use the terms client and user interchangeably. We also differentiate between two kinds of users when they execute our protocols: The key owner registers, updates, and audits her own key, while the other users request the key of some key owner from the providers. In our scenarios, hereafter, we assume that Alice is the key owner, and Bob is some other user who wants to communicate with Alice, hence, requests her key from the providers. Our model is depicted in Figure 1.

Adversarial model. We assume that the providers may be malicious, but not all providers collude (though some may). They may try to attack the integrity of the name-to-key bindings they store, or to equivocate and show different results for the same query coming from different users, while trying to be undetected. We assume honest behavior of the users only during the first key registration. But, they are not trusted for later key changes in the sense that they may later try to frame an honest provider. Our scheme provides non-repudiation for both the user and the provider, i.e., they cannot deny what they have already done, and helps find the origin of any inconsistency or misbehavior and provides cryptographic proofs to be used as evidence.

\subsection{Overview}

Public logs are proposed as an alternative to CAs that are not required to be trusted. Either client-side or server-side gossiping is used as the source of trust. The high level idea is that instead of trusting on only one entity, it is better to rely of a group of entities, hoping that not all of them misbehave simultaneously.

The existing solutions store keys of all users in an authenticated data structure, and hence, tie all keys together. This requires all users to check for their keys on any key registration/change. Instead, we store all keys of each user in a separate hash chain and remove the dependence between keys of different users. Each time a user registers a new key, the new key and the previous hash chain digest is hashed to obtain an new hash chain digest. Then, the home provider signs and shares this new digest with all other providers, i.e., we follow the server-side gossiping. This helps all providers keep the same view about the users.

To request a key, a user contacts a number of providers, and accepts the obtained key if they all provide the same view. She cannot rely on only one provider due to the possibility of equivocation.

\section{Key Authentication Service}

\subsection{Preliminaries}

Notation. We use || for concatenation, and $|X|$ to show the number of items in X. PPT means probabilistic polynomial time, and $\lambda$ is the security parameter. A function $v(\lambda): Z^{+} \rightarrow[0,1]$ is called negligible if $\forall$ positive polynomials $p, \exists$ a constant $c$ such that $\forall \lambda>c, v(\lambda)<1 / p(\lambda)$. Overwhelming probability is greater than or equal to $1-v(\lambda)$ for some negligible function $v(\lambda)$. By efficient algorithms, we mean those with expected running time polynomial in $\lambda$.

Interactive protocols. We present our key authentication scheme as a set of interactive protocols between stateful clients and stateful servers, instead of separately-executed algorithms. Each protocol may

\footnotetext{
${ }^{1} \mathrm{~A}$ client can be registered in multiple providers, but it complicates our discussion.
} 
receive inputs from both parties and may output some data at both parties. Hence, we represent a protocol as protocolName $\left(\right.$ out put $\left._{\text {client }}\right)\left(\right.$ out put $\left._{\text {server }}\right) \leftarrow\left(\right.$ input $\left._{\text {client }}\right)\left(\right.$ input $\left._{\text {server }}\right)$.

Hash functions are functions that take arbitrary-length strings, and output strings of some fixed length. Let $h: \mathcal{K} * \mathcal{M} \rightarrow \mathcal{C}$ be a family of hash functions, whose members are identified by $k \in \mathcal{K}$. A hash function family is collision resistant if $\forall P P T$ adversaries $\mathcal{A}, \exists$ a negligible function $v(\lambda)$ such that: $\operatorname{Pr}[k \leftarrow$ $\left.\mathcal{K} ;\left(x, x^{\prime}\right) \leftarrow \mathcal{A}(h, k):\left(x^{\prime} \neq x\right) \wedge\left(h_{k}(x)=h_{k}\left(x^{\prime}\right)\right)\right] \leq \vee(\lambda)$ for security parameter $\lambda$.

A signature scheme used for preserving message integrity, includes the following PPT algorithms [4]:

- $(s k, v k) \leftarrow \operatorname{Gen}\left(1^{\lambda}\right)$ : Generates a pair of signing and verification keys $(s k, v k)$ using the security parameter $\lambda$ as input.

- $\sigma \leftarrow \operatorname{Sign}(s k, m)$ : Generates a signature $\sigma$ on a message $m$ using the key $s k$.

- $\{$ accept $/$ reject $\} \leftarrow \operatorname{Verify}(v k, m, \sigma)$ : Checks whether $\sigma$ is a correct signature on a message $m$, using the verification key $v k$, and outputs an acceptance or a rejection signal, accordingly.

\subsection{Key Authentication Service Scheme}

Definition 2.1 (KAS scheme) A KAS scheme is composed of the following interactive protocols between a stateful user and a stateful provider:

- $\left(v k_{p}\right)\left(s k_{p}, v k_{p}\right) \leftarrow \operatorname{Setup}\left(1^{\lambda}\right)\left(1^{\lambda}\right)$. The provider starts up this protocol to generate the signing and verification keys $\left(s k_{p}, v k_{p}\right)$, given as input the security parameter $\lambda$, and shares the verification key with all users and providers. Essentially, we assume the providers' verification keys are part of some other well-established PKI.

- $\left(\sigma_{i}^{u}\right.$, accept /reject $)\left(r v k_{i-1}^{u}, v k_{i}^{u}, \sigma_{i}^{u}\right) \leftarrow \operatorname{Register}\left(i d_{u}, s k_{i-1}^{u}, s k_{i}^{u}, v k_{i}^{u}, v k_{p}\right)\left(s k_{p}, v k_{p}\right)$. The user registers/changes her key via a provider, given her ID $i d_{u}$, her previous key $s k_{i-1}^{u}$ (empty for the first time), and her new key pair $\left(s k_{i}^{u}, v k_{i}^{u}\right)$ as input. For a key change, she should provide a revocation statement $r v k_{i-1}^{u}$ signed with her previous signing key $s k_{i-1}^{u}$. The provider receives his key pair $\left(s k_{p}, v k_{p}\right)$ as input, stores user's key, computes a signature $\sigma_{i}^{u}$ on the result, and transfers it to the user. The user verifies it and outputs an acceptance or a rejection signal based on the result.

- $\left(v k_{i}^{u}\right.$, accept $/$ reject $)() \leftarrow \operatorname{Audit}\left(i d_{u},\left\{v k_{p}\right\}_{p \in \mathcal{P}}\right)\left(\right.$ inf $\left.o_{u}\right)$ : A user initiates this protocol to retrieve the (latest) key of a user $i d_{u}$. She specifies a subset of providers $P \subseteq \mathcal{P}$ at random and challenges them about the user $i d_{u}$. The providers send back their latest view about the user $i d_{u}$ given the user information info $o_{u}$. If all challenged providers gave the same answer, she accepts and outputs the received key $v k_{i}^{u}$ as the correct (latest) key of $i d_{u}$, and accept. Otherwise, she outputs $\perp$ and reject.

Both users and providers keep their own local states during execution of the protocols. Each user stores her latest key and the respective provider's signature (and the previous ones if she has enough resources). The providers also store all keys received from the users along with the signatures they have computed. The key owner runs Audit to see if the providers keep a correct view about her. If Audit returns accept and the obtained key matches the locally-stored key, she outputs accept. Otherwise, she outputs reject.

\subsection{Security Definitions of the Key Authentication Service}

Definition 2.2 (Correctness) considers the honest behavior of providers and users in a protocol execution. A key authentication scheme is correct if the following is satisfied with probability 1 over the randomness of the users and providers:

Each execution of the Audit protocol results in a key $v k_{u}$, which is the latest key registered by the key owner through the Register protocol. If such a user does not exist, it returns $\perp$.

Security game. If the providers deviate from honest behavior by providing different answers to the same query for different users, the users should detect this with high probability. The security game AuthGame $_{\mathcal{A}}(\lambda)$ between a challenger $\mathcal{C}$ who plays the role of all users (key owners and others) and all non-adversarial providers ${ }^{2}$, and the adversary $\mathcal{A}$ acting as the malicious providers, is defined as:

\footnotetext{
${ }^{2} \mathrm{We}$ assume that not all providers are colluding, there is at least one non-adversarial provider that the challenger will simulate.
} 
- Initialization. $\mathcal{C}$ runs the Setup protocol to initialize the environment on behalf of all non-adversarial providers, and shares their verification keys with $\mathcal{A}$. He stores the verification keys of malicious providers given by $\mathcal{A}$.

- Adaptively Chosen Queries. $\mathcal{A}$ asks the challenger to start a Register or an Audit protocol by providing the required information. For the Register protocol, he specifies a user and a provider that the user will register her key with. If the specified provider is a malicious one, $C$ acts as the user in the corresponding registration protocol with $\mathcal{A}$. He also stores the signatures coming from $\mathcal{A}$. Otherwise, $\mathcal{C}$ registers the key locally (acting as both the user and a non-adversarial provider) and shares the resulting signature with $\mathcal{A}$. For the Audit protocol, $\mathcal{A}$ requests a user ID, and $\mathcal{C}$ performs the associated audit and verifies the answer, and notifies $\mathcal{A}$ about the result. The queries can be repeated polynomially-many times, in any order, adaptively.

- Challenge. $\mathcal{A}$ specifies a user and sends an audit request to $\mathcal{C}$, who initiates an Audit protocol, giving the specified user ID as input. He outputs the obtained key and accept if Audit accepts, and $\perp$ and reject otherwise.

$\mathcal{A}$ wins the game if $\mathcal{C}$ accepts while the obtained key differs from the latest key $\mathcal{C}$ successfully registered on behalf of that user. The output of the game is defined to be 1 in this case.

Definition 2.3 ( $\varepsilon$-Security) A key authentication service scheme is $\varepsilon$-secure if $\operatorname{Pr}\left[\right.$ AuthGame $\left._{\mathcal{A}}(\lambda)=1\right] \leq \varepsilon$ for any efficient adversary $\mathcal{A}$.

\section{Construction}

Problems with Previous Proposals. Laurie et al. [7] builds an append-only Merkle hash tree and appends the new keys as they arrive. To be secure, after each new key registration/change, they distribute the root of the updated tree to the users who will gossip it among themselves to ensure that they all keep the same latest view of the keys registered in the log. This requires all users to connect and contribute in gossiping. In case of any partitioning among the users, the server can easily equivocate. In addition, all users need to verify the new tree. The key owner wants to check that her key is correctly registered/changed, while the other users want to make sure that they are not affected by this key registration/change. This poses an unacceptably heavy burden on users.

To alleviate the problem, CONIKS [9] uses server-side gossiping. Although this relieves the users from gossiping, they still need heavy and frequent verifications. CONIKS goes further and divides the time into epochs, accumulates all key registration/change requests during each epoch, and applies them all at the beginning of the next epoch. If $t$ key registration/change requests arrive in an epoch, instead of performing $t$ consistency checks, a client performs only one consistency check. However, this comes at the cost of giving outdated keys for users whose keys are changed in an epoch, until the beginning of the next epoch.

Our Observations and Solution. A problem common to all existing solutions $[6,7,9,12]$ is that they put all user-to-key bindings in a single data structure (i.e., a Merkle tree) on each provider. This ties all users together, and hence, forces all users to check whether or not any change in the data structure affects them, regardless of who initiated the change, which is a costly process. Instead, we store each user's data separately, preventing unwanted costly checks due to changes on other users. Even though on the surface it seems that we are gossiping more than just a root, the number of gossiping operations in our strategy is the same as in $[6,7,12]$, since one value is gossiped on every key change (although we can also gossip per epoch as in CONIKS, leaving some vulnerability window). However, they need to update the Merkle tree with $O(\log n)$ cost, while we only add a new value into the corresponding hash chain with only $O(1)$ cost.

Storing users' data separately brings other important advantages: the resulting scheme is perfectly privacy-preserving, since the proof for the a user's key does not contain any information about other owners (see Section 4.2). Moreover, there is no need for consistency checks, which are very costly operations in $[6,12]$. Even though CONIKS reduces the cost of this operation using epochs, the consistency check problem is inherent in tree-based approaches: on each key registration/change (or a group of them in CONIKS), 
Let $\mathrm{S}=\left(\right.$ Gen, Sign, Verify) be a secure signature scheme and $h:\{0,1\}^{*} \rightarrow\{0,1\}^{l}$ be a collision resistant hash function modeled as a random oracle. Construct a key authentication service scheme $K A S=($ Setup, Register, Audit) as:

- $\operatorname{Setup}\left(1^{\lambda}\right)\left(1^{\lambda}\right)$ :

- Each provider runs $\left(s k_{p}, v k_{p}\right)=\operatorname{S.Gen}\left(1^{\lambda}\right)$ and shares $v k_{p}$ with his users and the other providers.

- Each user stores $v k_{p}$ of her home provider.

- Register $\left(i d_{u}, s k_{i-1}^{u}, s k_{i}^{u}, v k_{i}^{u}, v k_{p}\right)\left(s k_{p}, v k_{p}\right)$ :

The key owner:

- If $i>1$, sets $r v k_{i-1}^{u}=\operatorname{S.Sign}\left(s k_{i-1}^{u}\right.$,'revocation statement')

- Sends $v k_{i}^{u}$ and $r v k_{i-1}^{u}$ to her home provider.

The home provider:

- Finds the last registered key, $v k_{i-1}^{u}$, and the last computed hash, $h_{i-1}^{u}$, for user $i d_{u}$.

- If S.Verify $\left(v k_{i-1}^{u}\right.$, 'revocation statement', $\left.r v k_{i-1}^{u}\right)==$ reject, outputs reject and exits.

- Otherwise, computes $h_{i}^{u}=h\left(i d_{u}\left\|h_{i-1}^{u}\right\| r v k_{i-1}^{u} \| v k_{i}^{u}\right)$ and $\sigma_{i}^{u}=\operatorname{S.Sign}\left(s k_{p}, h_{i}^{u}\right)$.

- Sends $h_{i}^{u}$ and $\sigma_{i}^{u}$ to the respective user and all other providers.

Other providers:

- Each other provider $p_{t}$ first checks if S.Verify $\left(v k_{p}, h_{i}^{u}, \sigma_{i}^{u}\right)==\operatorname{accept}$. If so, he computes $\sigma_{i}^{u, t}=\operatorname{S.Sign}\left(s k_{p_{t}}, h_{i}^{u}\right)$, stores $h_{i}^{u}$ and $\sigma_{i}^{u, t}$ locally, and sends $\sigma_{i}^{u, t}$ to the home provider.

The home provider:

- On receipt the acknowledgments $\sigma_{i}^{u, t}$, the home provider checks if S.Verify $\left(v k_{p_{t}}, h_{i}^{u}, \sigma_{i}^{u, t}\right)==$ accept for all other providers $p_{t}$. He stores the correct ones, and reveals the incorrect ones.

The key owner:

- The key owner checks if $h_{i}^{u}==h\left(i d_{u}\left\|h_{i-1}^{u}\right\| r v k_{i}^{u} \| v k_{i}^{u}\right)$ and S.Verify $\left(v k_{p}, h_{i}^{u}, \sigma_{i}^{u}\right)==\operatorname{accept}$. If so, she stores $h_{i}^{u}$ and $\sigma_{i}^{u}$ locally and outputs accept. Otherwise, she outputs reject and publishes misbehavior of the provider together with the corresponding $h_{i}^{u}$ and $\sigma_{i}^{u}$ as the proof, through public social networks.

- $\operatorname{Audit}\left(i d_{u},\left\{v k_{p}\right\}_{p \in \mathcal{P}}\right)\left(\right.$ info $\left._{u}\right)$ :

- The user selects a random subset of providers $P \subseteq \mathcal{P}$, and sends them the $\operatorname{Audit}\left(i d_{u}\right)$ request.

- The home provider replies with the latest information about the user being queried: $\left(h_{i-1}^{u}, r v k_{i-1}^{u}, v k_{i}^{u}, h_{i}^{u}\right)$.

- The other providers reply with the latest hash value, $h_{i}^{u, i_{t}}$, received from the home provider about that user.

- On receipt $\left(h_{i-1}^{u}, r v k_{i-1}^{u}, v k_{i}^{u}, h_{i}^{u}\right)$ from the home provider and $h_{i}^{u, i_{j}}$ from other providers, the user checks if:

* $h\left(i d_{u}\left\|h_{i-1}^{u}\right\| r v k_{i-1}^{u} \| v k_{i}^{u}\right)==h_{i}^{u}$. If not, misbehavior of the home provider is detected. She outputs $\perp$ and reject, publishes the misbehavior, and exits.

$* h_{i}^{u}==h_{i}^{u, i_{1}}==h_{i}^{u, i_{2}}==\ldots==h_{i}^{u, i_{t}}, \forall i_{j} \in P$. If so, she outputs $v k_{i}^{u}$ and accept. Otherwise, she outputs $\perp$ and reject, publishes the misbehavior, and starts the misbehaving detection process.

- (Owner Only) If the user audits for the key she owns, she also checks if the received key $v k_{i}^{u}$ is the one she last registered. If so, outputs accept. Otherwise, she uses it with the signature she received during registration, publishes the misbehavior case, outputs reject, and exits.

Figure 2: Construction of our key authentication service.

the provider updates the tree and distributes a new commitment. Then, all other owners should check the tree and ensure that their keys have not been changed.

Our KAS provides verifiable user-to-key binding service and consists of a number of providers each managing a subset of users. Each provider stores all registered keys of each user in its namespace in a separate hash chain. The provider, after adding the key into the respective chain, commits to (signs) the result, and sends it to the user for verification. Besides, he distributes (gossips) the last hash value in the chain and its signature to all other providers. To acknowledge the receipt, the other providers sign the received hash value, and return the signature to the initiating provider. Figure 2 shows our construction. 


\subsection{Description of the Operations}

Register. When a user registers her key for the first time, she provides the home provider with her id, $i d_{u}$, and her key, $v k_{1}^{u}$. (The user is assumed to be trusted for this operation.) The provider computes hash of this key as $h_{1}^{u}=h\left(i d_{u} \| v k_{1}^{u}\right)$, signs it as $\sigma_{1}^{u}=\operatorname{Sign}\left(s k_{p}, h_{1}^{u}\right)$, and returns back both values. When the user is registering a key change (i.e., the previous key, the $(i-1)^{t h}$ key, is revoked and she is providing a new one, the $i^{\text {th }}$ key), she should also prepare a revocation statement $r v k_{i-1}^{u}$ signed with her previous key and give it to the provider along with $i d_{u}$ and $v k_{i}^{u}$. On receipt, the provider verifies $r v k_{i-1}^{u}$, and if it is accepted, computes $h_{i}^{u}=h\left(i d_{u}\left\|h_{i-1}^{u}\right\| r v k_{i-1}^{u} \| v k_{i}^{u}\right)$ and $\sigma_{i}^{u}=\operatorname{Sign}\left(s k_{p}, h_{i}^{u}\right)$. In both cases, the provider returns $h_{i}^{u}$ and $\sigma_{i}^{u}$ to the user for verification. In addition, he distributes both values to all other providers, who will verify correctness of the signature and acknowledge by signing it if it is accepted. The home provider keeps the acknowledgments for detecting the source of later inconsistencies. The data structure for a key owner, Alice, on her home provider is a table as shown in Table 2. It contains all keys registered by Alice, all her key revocation statements, the home provider's signatures, and the other providers' acknowledgments.

Table 2: The data structure for a key owner, Alice, on her home provider.

\begin{tabular}{|l|c|c|c|c|}
\hline Alice's keys & $v k_{1}^{\text {Alice }}$ & $v k_{2}^{\text {Alice }}, r v k_{1}$ & $\ldots$ & $v k_{i}^{\text {Alice }, r v k_{i-1}}$ \\
\hline Provider's signatures & $h_{1}^{\text {Alice }}, \sigma_{1}^{\text {Alice }}$ & $h_{2}^{\text {Alice }}, \sigma_{2}^{\text {Alice }}$ & $\ldots$ & $h_{i}^{\text {Alice }}, \sigma_{i}^{\text {Alice }}$ \\
\hline$P_{2}$ 's acknowledgments & $\sigma_{1}^{\text {Alice }, 2}$ & $\sigma_{2}^{\text {Alice }, 2}$ & $\ldots$ & $\sigma_{i}^{\text {Alice }, 2}$ \\
\hline$\ldots$ & $\ldots$ & $\ldots$ & $\ldots$ & $\ldots$ \\
\hline$P_{m}$ 's acknowledgments & $\sigma_{1}^{\text {Alice }, m}$ & $\sigma_{2}^{\text {Alice }, m}$ & $\ldots$ & $\sigma_{i}^{\text {Alice }, m}$ \\
\hline
\end{tabular}

Each provider stores the hash values and signatures he receives about users of the other providers in a similar table, as presented in Table 3. The information in these tables are used for answering the audit queries, which if passed, ensure the auditing user that all providers have the same view about the queried user, meaning that there is no equivocation, with high probability. In other words, the providers help to make a web of trust through ensuring the auditing user that they all confirm binding of the provided key (hash value) to the queried user.

Table 3: The information provider $P_{t}$ keeps about Alice, a user of provider $P_{j}$.

\begin{tabular}{|l|c|c|l|c|}
\hline$P_{j}$ 's signatures & $h_{1}^{\text {Alice }}, \sigma_{1}^{\text {Alice }}$ & $h_{2}^{\text {Alice }}, \sigma_{2}^{\text {Alice }}$ & $\ldots$ & $h_{i}^{\text {Alice }}, \boldsymbol{\sigma}_{i}^{\text {Alice }}$ \\
\hline$P_{t}$ 's acknowledgments & $\sigma_{1}^{\text {Alice }, t}$ & $\sigma_{2}^{\text {Alice }, t}$ & $\ldots$ & $\sigma_{i}^{\text {Alice }, t}$ \\
\hline
\end{tabular}

On receipt the answer, the key owner verifies the received signature to see if the home provider has correctly registered her key. She can compute either $h_{1}^{\prime u}=h\left(i d_{u} \| v k_{1}^{u}\right)$ or $h_{i}^{\prime u}=h\left(i d_{u}\left\|h_{i-1}^{u}\right\| r v k_{i-1}^{u} \| v k_{i}^{u}\right)($ she knows all required information) and compare it with what received from the home provider: $h_{1}^{\prime u}==h_{1}^{u}$ for the first key registration or $h_{i}^{\prime} u==h_{i}^{u}$ for the subsequent key changes. If the test passed, she verifies the signature. If it is verified successfully too, she ensures that her key is correctly registered. Then, she can run an Audit protocol to see if the key registration is correctly reflected on the other providers.

Audit. From time to time, the key owner, Alice, checks if the providers keep storing her key and hash value intact. This is because other users cannot check authenticity of her keys. Hence, they rely on regular checks of Alice. If Alice detects equivocation or any other misbehavior, in addition to going to arbitration, she should somehow inform the other users about the misbehavior (e.g., via publishing in social networks [9]) and prevent them from using the fake or outdated keys registered in her name.

When another user, Bob, wants to communicate with Alice, he asks for Alice's key from the providers. If Bob requests Alice's key only from her home provider, the home provider may equivocate and give a fake or an outdated key. He needs to make sure, with high probability, that he retrieves a genuine key. Moreover, prior to obtaining the key, he should first check and ensure that there is no equivocation report by Alice.

Bob selects a random subset $P$ of providers $P$, and sends them the audit request. They return the last hash value they have received from the home provider. Bob also contacts Alice's home provider, who 
returns the last registered key, as well as information required to recompute the last hash in the chain. Bob then checks whether all received hash values are the same (otherwise an equivocation is detected). He also re-hashes the values provided by Alice's home provider. If everything verifies, he returns the obtained key and accept. Otherwise, he outputs $\perp$ and reject, and starts the misbehavior detection process.

Alice, as the owner of the key, in addition, checks whether the obtained key matches the one she already registered. If so, she outputs accept, and reject otherwise. In case of any mismatch, Alice announces the misbehavior through the social networks with the home provider's signature received during the registration as the proof. The misbehavior detection probabilities are discussed in more detail in Appendix A. Essentially, we obtain similar probabilities as in CONIKS [9].

Misbehavior detection. When the key owner or another user discovers a misbehavior, she can trace and find the source of that misbehavior. This is due to the non-repudiation property of our scheme. Remember that the key is distributed by the home provider only, and the other providers distribute the hash values. Therefore, if the given key does not match the given hash value (all users can detect it), this can be used as the witness of home provider's misbehavior. If the given key matches the given hash value but it is not the genuine key (only the key owner can detect it), the home provider is misbehaving, and the key owner can use these values together with the home provider's signature received during the registration as the cryptographic proof of the misbehavior. However, she cannot claim that a given key is fake if she has already registered the key. The home provider can show this case using the signed revocation statement he received from the key owner.

Whenever the received hash values does not match each other (all users can detect it), a subset of providers are equivocating. The user (or any authority) asks the home provider acknowledgments of the other challenged providers, and asks the other challenged providers the signature they have received from the home provider. Verifying the signatures will reveal the misbehaving provider(s).

\section{Analysis}

\subsection{KAS Security Proof}

Correctness follows from the correctness of the hash function used by the providers. If the key owner has already been registered, the hash function guarantees that whenever her key is requested, the requesting party receives the correct key. If not, $\perp$ together with non-membership proof will be returned.

Theorem 4.1 Our KAS scheme is secure according to Definition 2.3 provided that the underlying hash function is collision-resistant.

Proof 4.1 We reduce the security of our KAS scheme to that of the underlying hash function. If a PPT adversary $\mathcal{A}$ wins the security game of our KAS scheme with non-negligible probability, we use it to construct another PPT algorithm $\mathcal{B}$ who breaks collision-resistance of the hash function, with non-negligible probability. $\mathcal{B}$ acts as the adversary in the hash function security game with the challenger $\mathcal{C}$. In parallel, $\mathcal{B}$ plays the role of the challenger in the KAS game with $\mathcal{A}$.

Initialization. $\mathcal{B}$ receives a hash function $h($.$) from \mathcal{C}$, and shares it with $\mathcal{A}$. The signature keys are generated and shared.

Adaptively Chosen Queries. $\mathcal{A}$ sends $\mathcal{B}$ a request to start a protocol, with the required information:

- For a Register protocol, a user ID, $i d_{u}$, and a provider ID are given. $\mathcal{B}$ generates key pair $\left(s k_{i}^{u}, v k_{i}^{u}\right)$ and the revocation statement $r v k_{i-1}$ for the given user and stores them locally. If the given provider is non-adversarial, $\mathcal{B}$ computes $h_{i}^{u}=h\left(i d_{u}\left\|h_{i-1}^{u}\right\| r v k_{i-1}^{u} \| v k_{i}^{u}\right)$, signs it as $\sigma_{i}^{u}$, stores $h_{i}^{u}$ and $\sigma_{i}^{u}$ locally, sends them to $\mathcal{A}$, and keeps the acknowledgments coming back. If the given provider is malicious, $\mathcal{B}$ asks $\mathcal{A}$ to register the key for the user, and stores and acknowledges the potential hashes and signatures coming back.

- For an Audit protocol about $i d_{u}$, B selects a random subset of providers $P \subseteq \mathcal{P}$, as well as the user's home provider, and challenges them about $i d_{u}$. For those challenged providers who are non- 
adversarial, $\mathcal{B}$ himself provides the answer, while the answer of the malicious ones are given by $\mathcal{A}$. On receipt all answers, $\mathcal{B}$ performs the verification as in the protocol. He informs $\mathcal{A}$ about the result.

Challenge. The adversary specifies a user $i d_{u}$ and asks $\mathcal{B}$ to challenge him about this user. $\mathcal{B}$ runs the Audit protocol as described. If $\mathcal{B}$ accepts $\mathcal{A}$ 's answer while the obtained key disagrees with his local knowledge, we consider two cases:

- The returned hash value(s) is the same as the locally-stored one. In this case, a collision is found. If $\mathcal{B}$ accepts $\mathcal{A}$ 's answer with probability $\varepsilon_{1}$, we can use it to break security of the hash function with probability $\varepsilon_{1}$. Since the hash function is collision-resistant by assumption, $\varepsilon_{1}$ must be negligible, which means that $\mathcal{A}$ has negligible chance to pass $\mathcal{B}$ 's verification successfully and win the game. Hence, our KAS scheme is secure in this regard assuming that the underlying hash function is collision-resistant.

- The returned hash values differ from the locally-stored one: that is an indication of an equivocation. The equivocation of providers is discussed in more detail in Appendix A. According to the discussion, we can approximate the provider equivocation probability with $(1-f)^{k}$ against the key owner and $f^{k}$ against the other users, where $f$ is the fraction of providers distributing fake hash values, and $k=|P|$ is the size of the challenged subset of providers.

Overall, the discussion reveals that when $f$ is large, the key owner will detect the misbehavior with high probability. When $f=1 / 2$, the other users have a higher probability of misbehavior detection. The figures in Appendices A.1 and A.2 confirm this statement. Moreover, they will detect the equivocation with probability $1-\left(f-f^{2}\right)^{k}$ as discussed in Appendix A.3.

Hence, our scheme is $\left(f-f^{2}\right)^{k}$-equivocal, which means that the equivocation detection probability increases very fast as we increase $k$. This is maximized when $f=1 / 2$. Interestingly, increasing $f$ (from $1 / 2$ ) decreases the detection probability (except if $f=1$ where all providers are colluding).

- When $f$ is very small, the probability of misbehavior detection by Alice is not very high (e.g., when $f=0.01, k=50$ leads to detection probability $\left.1-(1-0.01)^{50}=0.395\right)$. But, this means that the Audit protocol will select all honest providers for audit with high probability, and hence, the obtained key will be the correct key with high probability.

- When $f$ is close to 1 , the key owner's probability of misbehavior detection is very high (e.g., when $f=0.99, k=50$ leads to detection probability $1-(1-0.99)^{50}$, which is almost 1$)$. Note that in this case, the other users' probability of misbehavior detection is not high, i.e., 0.395. In other words, they will accept the given (fake) key with probability 0.605. But, in this case, the key owner can detect the equivocation with probability almost 1 and notify the other users.

- These computations show only the results of one audit. But, the key owner audits regularly over the time. If the Audit protocol selects the providers uniformly, it is expected that after $|\mathcal{P}| /|P|$ audits, all providers will be challenged at least once. This means that the key owner will eventually detect the misbehavior.

- To obtain this detection probability, we do not need all the providers to be home providers. Essentially, maybe only a few providers actually store the keys and have registered users, but other providers just participate in the gossip of the hash values. This means, we can add such independent 'dummy' providers to the system to increase overall security. This is expected to ease the deployment of our system.

To sum up, our proposed KAS scheme is $\varepsilon=\left(\varepsilon_{1}, \varepsilon_{2}\right)$-secure, i.e., the adversary can break it with probability $\varepsilon_{1}$, which is negligible in the security parameter if the underlying hash function is secure, and it is $\left(\varepsilon_{2}=\left(f-f^{2}\right)^{k}\right)$-equivocal meaning that the adversary can equivocate with a very small probability.

\subsection{Discussion}

Privacy-preserving means that auditing or requesting a user's key should not reveal any information about the other users. The existing schemes [7, 12,9] generate a proof using (encoded) information of some other 
users (i.e., the path from the leaf node storing the requested key to the root, in a Merkle tree). This means that privacy of other users is not fully preserved and one can gain some information from the proof about other users [9]. Our scheme stores keys of each user separately, and the proofs are only based on her own data. Therefore, it reveals nothing about the other users.

No need for a consistency proof. In a tree-based approach where all users' data are tied together, a key registration/change updates the tree. Hence, all other users should check that they have not been affected in an unwanted manner. They receive a proof on each change (or on the beginning of each epoch in CONIKS), and verify it. Considering that the proof is of size $O(\log n)$, where $n$ is the number of registered users, and there are $r$ key registrations/changes per day, each client receives and verifies proofs of size $O(r \log n)$ per day. But, there is no consistency check in our KAS as the users' data are stored separately.

Proof of absence. On receipt of a key request, if a key has already been registered for the requested user, the home provider returns the registered key and his signature on the key, as the proof of registration. The proof serves as the proof of presence. On the other hand, if the requested user has no registered key, our scheme returns $\perp$, which servers as the proof of absence. This is a result of equation detection in our schemes: if it returns $\perp$ for a registered user, this will be caught with high probability.

Non-repudiation. A common problem among the existing schemes is that one cannot show the origin of any potential inconsistency. In our KAS scheme, each party commits to all her work or acknowledges others', and stores commitments or acknowledgments form other parties during the key registration/change. The key owner commits to all her key changes via revocation statements (following the first key). The home provider also commits to all keys he registers for his users. The other providers also acknowledge all signatures received form the home providers. Therefore, no party can later deny his work during the key registration protocol.

Moreover, in case of any misbehavior, it is easy to track and find the source of that misbehavior. Then, one can use it as a cryptographic proof about the misbehavior, and take the specified actions, i.e., going to arbitration or publishing it through social networks to inform others.

\subsection{Asymptotic Comparison to Previous Work}

We compare operation complexities of our KAS against the existing schemes. They all use gossiping as a means of providing trust. The schemes given in $[6,12]$ use client-side gossiping, while CONIKS [9] and ours use server-side gossiping. The comparisons show the case where a single or a constant number of operations are performed.

Key registration/change. Since all users' data are tied together in the existing schemes, a key registration/change (or an epoch in CONIKS) forces the provider to generate proofs of size $O(\log n)$ for all users and send them to the respective users (consistency proofs). This requires total $O(n \log n) \operatorname{computation}$ and communication cost at the provider, which are not taken into account in the previous work. This is an $O(1)$ operation in our KAS since the provider performs a constant number of operations and distributes constant-size proofs over the network on each update. However, the key owners of KAS audit the providers once in a time, roughly like an epoch in CONIKS. The providers reply only with the latest keys and hash values without any computation, which accounts for $O(n)$ server computation and communication, in total.

Gossiping. In CONIKS and our scheme, there is a gossiping at the provider side, whereas in the other two schemes gossiping is done at the client side. Note that in practice, we expect maybe only hundreds of providers whereas many millions of clients will take place in the system. Table 4 presents the comparison, showing an $O(\log n)$ decrease in all operations in our KAS.

Key revocation. The scheme given in [6] has no an efficient way for key revocation, hence, it needs to traverse the whole ADS with cost $O(n)$. But, the two others [12,9] can do it with $O(\log n)$ time and space complexity, i.e., the server should generate an $O(\log n)$ proof for each user (summing up to $O(n \log n)$ communication) in $O(n \log n)$ time. This is also an $O(1)$ operation in our scheme since there is no correlation between data of different users. 
Table 4: A comparison of key registration/change costs. $n$ is the number of users.

\begin{tabular}{|l|c|c|c|c|c|}
\hline \multirow{2}{*}{ Scheme } & \multicolumn{2}{|c|}{ Provider } & \multicolumn{2}{|c|}{ User } & \multirow{2}{*}{ Gossiping } \\
\cline { 2 - 5 } & Key Reg. & Proof Gen. & Comp. & Proof Size & \\
\hline Laurie et al. [6] & $O(\log n)$ & $O(n \log n)$ & $O(\log n)$ & $O(\log n)$ & Client-side \\
\hline ECT [12] & $O(\log n)$ & $O(n \log n)$ & $O(\log n)$ & $O(\log n)$ & Client-side \\
\hline CONIKS [9] & $O(\log n)$ & $O(n \log n)$ & $O(\log n)$ & $O(\log n)$ & Server-side \\
\hline Our KAS & $O(1)$ & $O(1)(O(n)$ audits) & $O(1)$ & $O(1)$ & Server-side \\
\hline
\end{tabular}

Audit. This operation is only needed in CONIKS and our scheme. Similarly, it requires $O(n \log n)$ and $O(n)$ server computation and communication in CONIKS and KAS, respectively. Moreover, the client computation and proof size are $O(\log n)$ and $O(1)$ in CONIKS and KAS, respectively.

\subsection{Performance Analysis}

Setup. To evaluate our scheme, we implemented a prototype using the Cashlib library [8]. All experiments were performed on a $2.50 \mathrm{GHz}$ machine with 24 cores (using a single core), with $16 \mathrm{~GB}$ RAM and Ubuntu 12.04 LTS operating system, hosting all our providers. Hence, the inter-provider communications were performed as loopback. The clients were deployed on a dualcore $2.5 \mathrm{GHz}$ laptop machine with $4 \mathrm{~GB}$ RAM, running Ubuntu 14.04 LTS, connected to the same LAN. We used settings similar to CONIKS: each provider has $n=10 \mathrm{M}$ registered users, and a user changes her key once a year, which accounts for $\sim 27,400$ changes per day. The security parameter $\lambda=128$, and we use SHA-256 as the hash function and the DSA signature scheme with key pair size $(2048,256)$ bits according to [3]. The numbers are averages of 50 runs.

Key registration/change. The client sends the home provider her new key (and revocation statement of the previous key), which has a fixed size. The provider computes hash of the key (32 B), signs the result (64 B), and shares the hash values and the signature with other providers, as well as the key owner. Therefore, the inter-provider communication is $96 \mathrm{~KB}$ in total when $|\mathcal{P}|=1000$, and the provider-user communication is only $96 \mathrm{~B}$. This happens for each client once per key lifetime, not per epoch as in CONIKS.

The previous schemes require the provider to generate and send a consistency proof to each user on each key registration/change [6,12] (each epoch in CONIKS [9]). Considering that the proof is of size $O(\log n)$, where $n$ is the number of users, and there are $r$ key registrations/changes per day, each client receives and verifies $O(r \log n)$ proofs per day. This is $\sim 800 \mathrm{MB}$ (for $10^{9}$ users) in ECT and $100 \mathrm{~KB}$ (for $10^{7}$ users) using the compressed proofs in CONIKS, per day.

The $O(n \log n)$ proof that the provider prepares and distributes over the network each time is more than $3.11 \mathrm{~GB}$ in total in CONIKS. Assuming 27,400 key changes and 288 epochs per day, the providers prepare and distribute more than 895 GB proof per day in CONIKS. The others $[6,12]$ perform even worse. In our scheme, the providers perform a constant number of operations and distributes constant-size proofs, leading to a total of $\sim 2.5 \mathrm{MB}$ of proof per day. This is a great out-performance over the existing schemes.

Computation time. It takes $\sim 0.5 \mathrm{~ms}$ for the provider in KAS to perform the key registration and send back the proof, while it takes $\sim 2.6 s$ in CONIKS.

Audit. Assuming the key owners perform audit operations once in a time equal to a CONIKS epoch (i.e., 5 minutes), the providers return the already-computed hash values (and signatures in previous schemes), without any computation. During the normal operation, our providers respond with the latest hash values only. Assuming the challenge size $k=50$, each user receives a response of size $\sim 1.56 \mathrm{~KB}$ in our scheme, and $\sim 4.68 \mathrm{~KB}$ in CONIKS, for each audit. These sum up to $\sim 450 \mathrm{~KB}$ in our scheme, and $\sim 1.31 \mathrm{MB}$ in CONIKS, per day. For the providers, this is an $O(n)$ operation, which sums to $\sim 305 \mathrm{MB}$ of proof for one round of audit, and $\sim 85$ GB per day, in our scheme. While CONIKS will transfer $\sim 915 \mathrm{MB}$ of proof for one round of audit, and $\sim 257.5$ GB per day. Our KAS saves more than $67 \%$ of the daily communication compared to CONIKS. The results are shown in Table 5. 
Table 5: A comparison of audit proof sizes in our KAS and CONIKS, $k=50$.

\begin{tabular}{|l|c|c|c|c|c|c|}
\hline \multirow{2}{*}{ Scheme } & \multicolumn{3}{|c|}{ Provider } & \multicolumn{3}{c|}{ User } \\
\cline { 2 - 7 } & Complexity & One epoch & Per day & Complexity & One Audit & Per day \\
\hline CONIKS & $O(n \log n)$ & $915 \mathrm{MB}$ & $257.5 \mathrm{~GB}$ & $O(1)$ & $4.68 \mathrm{~KB}$ & $1.31 \mathrm{MB}$ \\
\hline Our KAS & $O(n)$ & $305 \mathrm{MB}$ & $85 \mathrm{~GB}$ & $O(1)$ & $1.56 \mathrm{~KB}$ & $450 \mathrm{~KB}$ \\
\hline
\end{tabular}

\section{References}

[1] A. Barenghi, M. Beretta, A. D. Federico, and G. Pelosi. Snake: An end-to-end encrypted online social network. In High Performance Computing and Communications, pages 763-770. IEEE, 2014.

[2] W. Diffie and M. E. Hellman. New directions in cryptography. Information Theory, IEEE Transactions on, 22(6):644-654, 1976.

[3] P. Gallagher and C. Kerry. Digital signature standard (dss). NIST, 2013. FIPS PUB 186-4.

[4] S. Goldwasser, S. Micali, and R. L. Rivest. A digital signature scheme secure against adaptive chosenmessage attacks. SIAM, 17(2):281-308, 1988.

[5] G. Greenwald and E. MacAskill. Nsa prism program taps in to user data of apple, google and others. http://www.theguardian.com/world/2013/ jun/06/us-tech-giants-nsa-data, 2013. Accessed: 26/03/2015.

[6] B. Laurie and E. Kasper. Revocation transparency. Google Research, 2012.

[7] B. Laurie, A. Langley, and E. Kasper. Rfc 6962: Certificate transparency, 2013.

[8] S. Meiklejohn, C. Erway, A. Küpçü, T. Hinkle, and A. Lysyanskaya. Zkpdl: A language-based system for efficient zero-knowledge proofs and electronic cash. In USENIX Security Symposium, 2010.

[9] M. S. Melara, A. Blankstein, J. Bonneau, M. J. Freedman, and E. W. Felten. Coniks: A privacypreserving consistent key service for secure end-to-end communication. 2014.

[10] M. Naor and K. Nissim. Certificate revocation and certificate update. Selected Areas in Communications, IEEE Journal on, 18(4):561-570, 2000.

[11] R. L. Rivest, A. Shamir, and L. Adleman. A method for obtaining digital signatures and public-key cryptosystems. Communications of the ACM, 21(2):120-126, 1978.

[12] M. D. Ryan. Enhanced certificate transparency and end-to-end encrypted mail. Proceedings of NDSS. The Internet Society, 2014.

[13] S. G. Weber. Enabling end-to-end secure communication with anonymous and mobile receivers - an attribute-based messaging approach. Cryptology ePrint Archive, Report 2013/478, 2013.

[14] D. Wendlandt, D. G. Andersen, and A. Perrig. Perspectives: Improving ssh-style host authentication with multi-path probing. In USENIX, pages 321-334, 2008. 


\section{A Equivocation Detection}

Setup. Alice is the key owner that knows her (latest) key and signature. Bob is another user who wants to obtain Alice's key. We assume that there are 1000 providers (i.e., $m=|\mathcal{P}|=1000$ ), of which $k$ are selected randomly and challenged each time. $e$ portion of providers are equivocating, i.e., they give the correct signatures to Alice while giving fake signatures (about Alice) to other users and $f$ portion of other providers. We consider misbehavior detection by either the key owner or other users and equivocation detection separately.

\section{A.1 Misbehavior Detection by Owner}

These tests are done by the key owner, Alice. Since there is no trusted party in the scheme, Alice should regularly audit and make sure all providers maintain the same view about her. Since the audit is done regularly, each one verifying a random subset of providers, Alice will detect any misbehavior over the time, with high probability. We consider two scenarios:

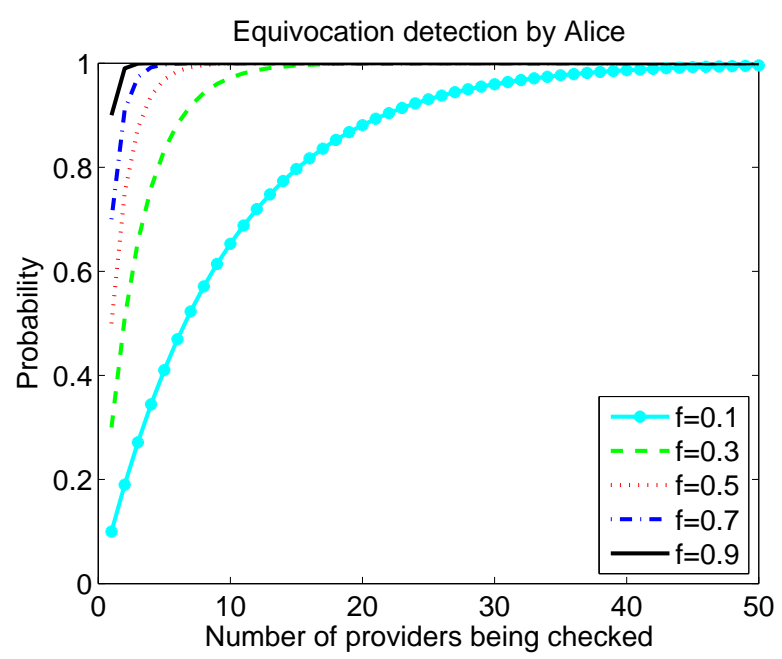

(a) First scenario.

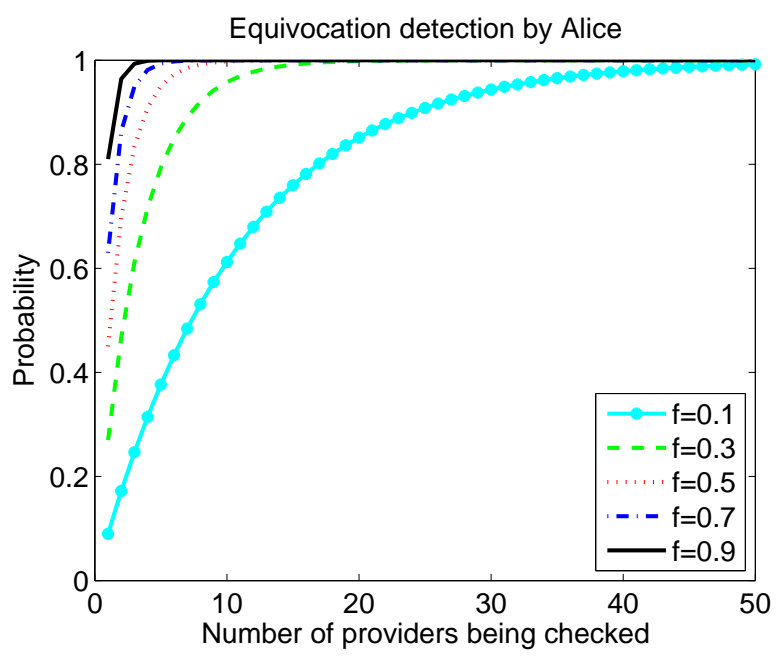

(b) Second scenario, $e=0.1$.

Figure 3: Misbehavior detection by Alice.

First scenario: only Alice's home provider is equivocating. The home provider gives the correct key and hash value, while $f$ portion of providers will give fake hash value. With probability $\Pi_{i=0}^{k-1} \frac{(1-f) m-i}{m-i}$, all providers selected for challenge are from the set of those that are given correct hash values and the home provider, in which case, there is no misbehavior. After converting the probability formula to $\Pi_{i=0}^{k-1}\left(1-\frac{f m}{m-i}\right)$ and a variable change, we can approximate it with $(1-f)^{k}$, leading to misbehavior detection probability $1-(1-f)^{k}$. The probability she detects an equivocation is then $1-\Pi_{i=0}^{k-1} \frac{(1-f) m-i}{m-i} \simeq 1-(1-f)^{k}$. This is represented in Figure $3 \mathrm{a}$ for different values of $k$ and $f$. It shows that the bigger the portion of providers receiving and distributing fake keys and hash values (the larger value of $f$ ), the less the probability of misbehavior, and the bigger the misbehavior detection probability. This is because the probability of auditing misbehaving providers increases. For example, when $f=0.5$ (i.e., $50 \%$ of providers are distributing fake values), with $k=20$ and $k=50$, Alice can detect the misbehavior with probability $0.999,999$ and $0.999,999,999,999,999$, respectively.

Second scenario: $\boldsymbol{e}$ portion of providers are equivocating. Similarly, Alice can detect misbehavior with probability $1-\Pi_{i=0}^{k-1} \frac{(e+(1-e)(1-f)) m-i}{m-i}$, which can be approximated as above by $1-(1-f(1-e))^{k}$. This is represented for different values of $e$ in Figures $3 \mathrm{~b}(e=0.1), 4 \mathrm{a}(e=0.5)$, and $4 \mathrm{~b}(e=0.9)$. These figures show that the more the portion of equivocating providers, the less the probability of misbehavior detection. This is due to the fact that the equivocating providers give genuine hash values (and keys) to Alice. 


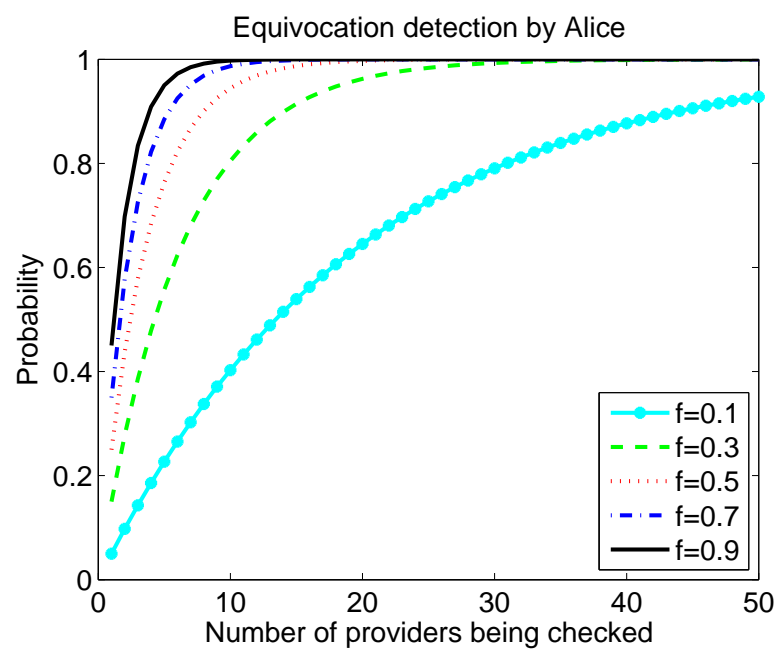

(a) $e=0.5$.

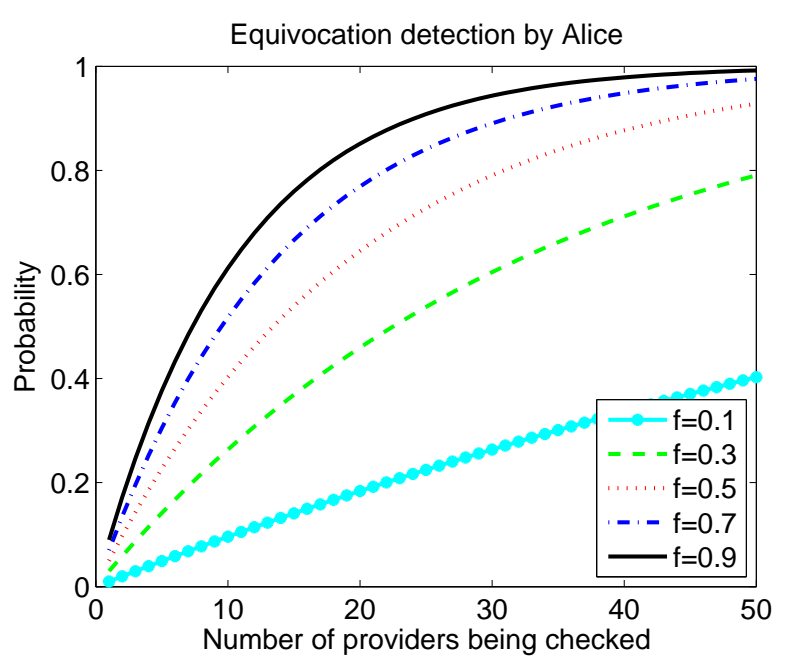

(b) $e=0.9$.

Figure 4: Equivocation detection by Alice, second scenario.

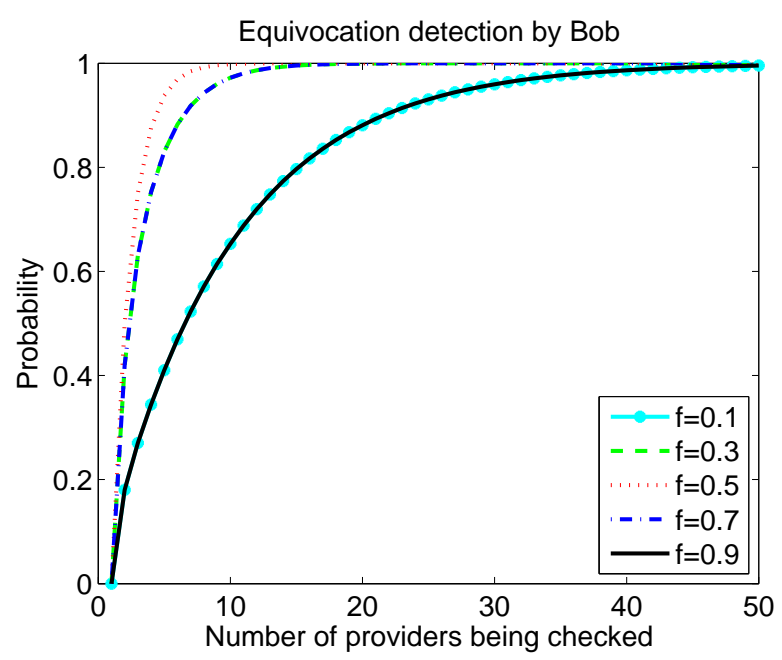

(a) First scenario.

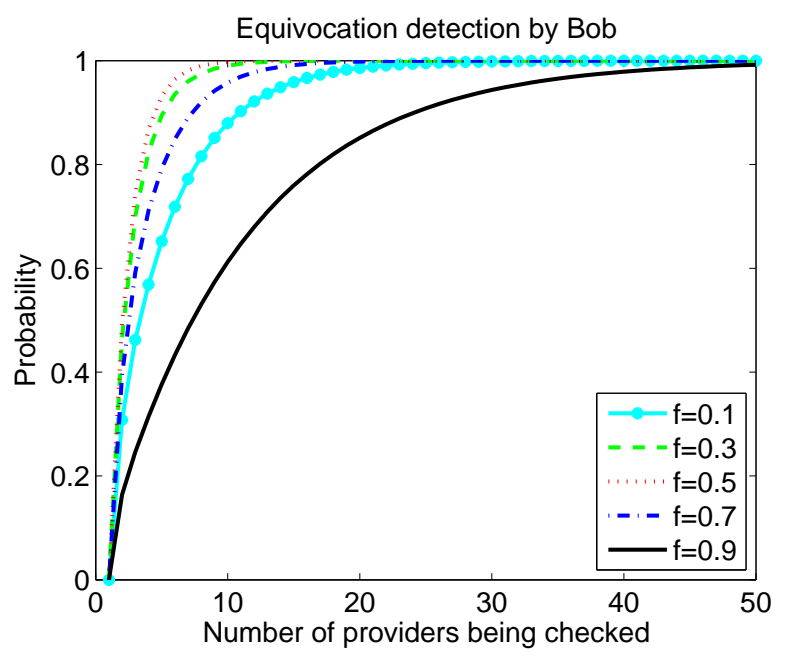

(b) Second scenario, $e=0.1$.

Figure 5: Misbehavior detection by Bob.

\section{A.2 Misbehavior Detection by Other Users}

These tests are done by Bob who is trying to obtain Alice's key. To make sure the obtained key is a genuine key, he checks whether a randomly-selected subset of providers give the same view about Alice, hoping she would have already detected and published any inconsistency about her. We investigate two scenarios:

First scenario: only Alice's home provider is equivocating. He returns the correct key to Alice, but fake key and hash value (for Alice) to Bob. Moreover, he gives $f$ portion of the other providers fake hash value for Alice, and $1-f$ portion of them the genuine hash value of Alice. Bob receives the genuine hash value (and key) from all challenged providers with probability $\Pi_{i=0}^{k-1} \frac{(1-f) m-i}{m-i}$ (that can be approximated by $\left.(1-f)^{k}\right)$ and a fake hash value (and key) from all challenged providers with probability $\Pi_{i=0}^{k-1} \frac{m f-i}{m-i}$ (that can be approximated by $\left.f^{k}\right)$. Hence, he can detect a misbehavior with probability $1-\Pi_{i=0}^{k-1} \frac{(1-f) m-i}{m-i}-\prod_{i=0}^{k-1} \frac{m f-i}{m-i}$ $\left(\simeq 1-(1-f)^{k}-f^{k}\right)$, as depicted in Figure 5a for different values of $k$ and $f$. An interesting fact is that when either $f=a$ or $f=1-a$, where $0 \leq a \leq 1$, the probability of misbehavior detection is the same. The explanation is that when a small portion of providers are distributing fake hash values, Bob will receive all genuine hash values with high probability. But when a big portion of providers are distributing fake hash values, Bob will receive all fake hash values with high probability, and will accept it. Therefore, the 


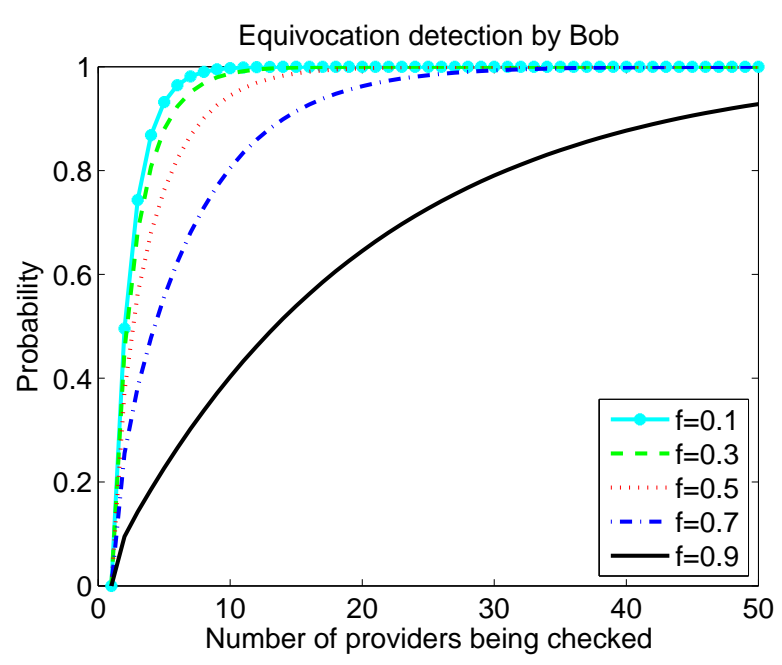

(a) $e=0.5$.

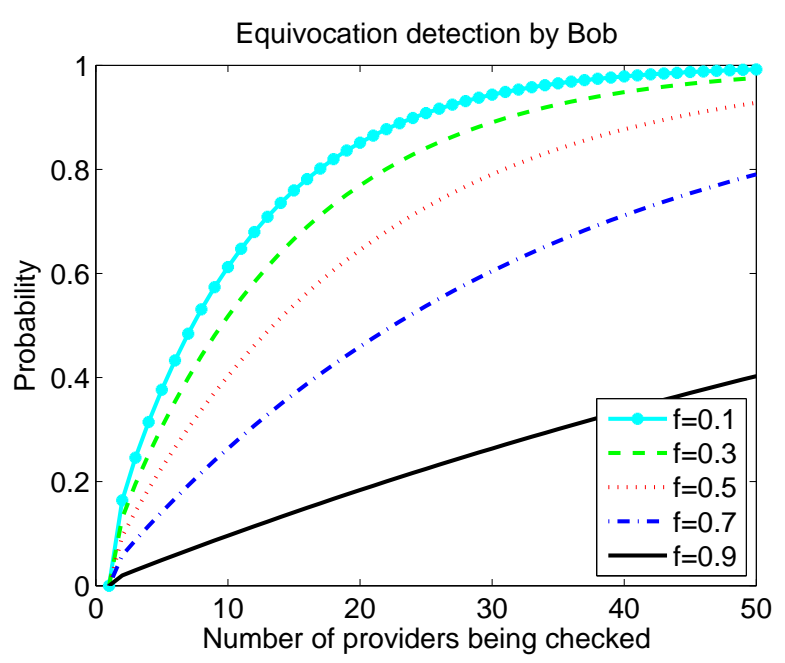

(b) $e=0.9$.

Figure 6: Misbehavior detection by Bob, second scenario.

probability of misbehavior detection is small. When $f=0.5$, both the providers distributing fake and genuine hash values will be selected with high probability, and hence the probability of misbehavior detection is high. In this case, with $k=10, k=20$ and $k=50$, Bob can detect the misbehavior with probability 0.998 , $0.999,998$ and $0.999,999,999,999,998$, respectively.

Second scenario: $\boldsymbol{e}$ portion of providers are equivocating. They give fake hash values and keys (for Alice) to Bob and $f$ portion of non-equivocating (i.e., $(1-e)$ portion of) providers, and the real hash values (and keys) to the remaining ones. Bob, similarly, can detect equivocation only if he receives different hash values for Alice in the same query. $e+(1-e) f$ portion of providers will all give fake hash values, while $(1-e)(1-f)$ portion of them will return genuine hash values. Hence, Bob can detect misbehavior with (approximated) probability $1-\Pi_{i=0}^{k-1} \frac{(e+(1-e)(1-f)) m-i}{m-i}-\Pi_{i=0}^{k-1} \frac{((1-e) f) m-i}{m-i}$ (that can be approximated by $\left.1-(1-(1-e) f)^{k}-((1-e) f)^{k}\right)$. This is represented for different values of $e, f$, and $k$ in Figures $5 b$ $(e=0.1), 6 \mathrm{a}(e=0.5)$, and $6 \mathrm{~b}(e=0.9)$. These figures show that when $e$ is fixed, the probability of misbehavior detection decreases as $f$ increases. This is for the fact that more and more providers help equivocating providers by giving fake hash values that makes the detection hard. The same fact holds as $e$ increases. These figures state that, in general, Bob does not have a good chance of misbehavior detection by himself. But, since Alice has a very high probability of misbehavior detection, she will help Bob in this regard.

\section{A.3 Equivocation Detection}

An equivocation occurs if 1) the key owner receives and accepts the correct key and 2) another user receives and accepts a fake key. This means that the providers successfully gave a fake key for Alice to Bob while Alice is regularly checking her key. We consider two scenarios:

First scenario: only Alice's home provider is equivocating. Using the above computations, Alice accepts the obtained (correct) key with probability $(1-f)^{k}$. Moreover, Bob will accept a fake key with probability $f^{k}$. Since these two events need to happen together, an equivocation occurs with probability $f^{k}(1-f)^{k}=\left(f-f^{2}\right)^{k}$, and the probability of detection is $1-\left(f-f^{2}\right)^{k}$. This is represented in Figure 7 a for different values of $k$ and $f$. It shows that when $f=1 / 2$, the equivocation detection probability is minimum, and it increases vary fast as $f$ approaches either 0 or 1 . When $f=0.3$ or $f=0.7$, with $k=10$ and $k=20$, the equivocation detection probability is $0.999,999,833$ and $0.999,999,999,999,972$, respectively.

Second scenario: $\boldsymbol{e}$ portion of providers are equivocating. Similarly, Alice will accept the correct key with probability $(e+(1-e)(1-f))^{k}$ and Bob accepts a fake key with probability $(e+(1-e) f)^{k}$. This leads to the equivocation detection probability $1-((e+(1-e) f)(e+(1-e)(1-f)))^{k}$, which is represented for different values of $e$ and $f$ in Figures $7 \mathrm{~b}(e=0.1), 8 \mathrm{a}(e=0.5)$, and $8 \mathrm{~b}(e=0.9)$. These figures interestingly show 


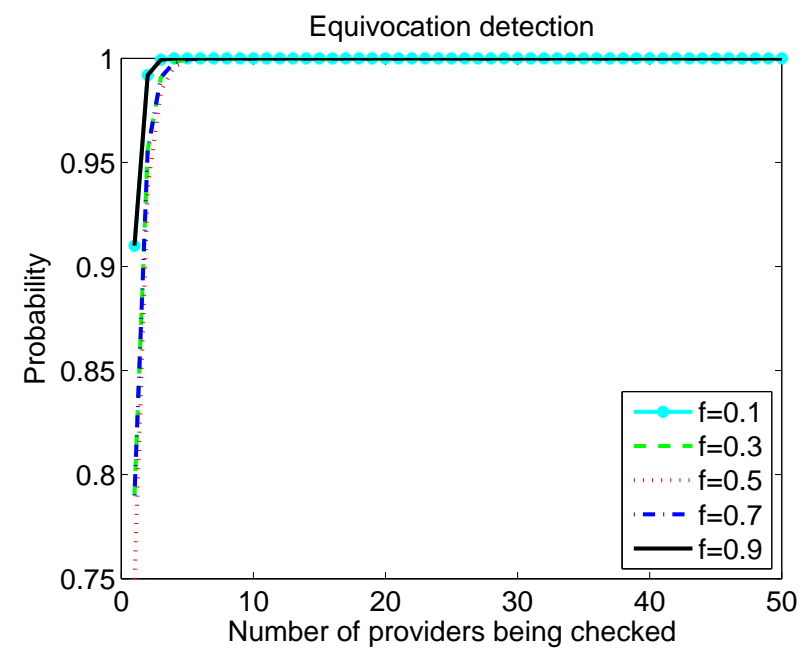

(a) First scenario.

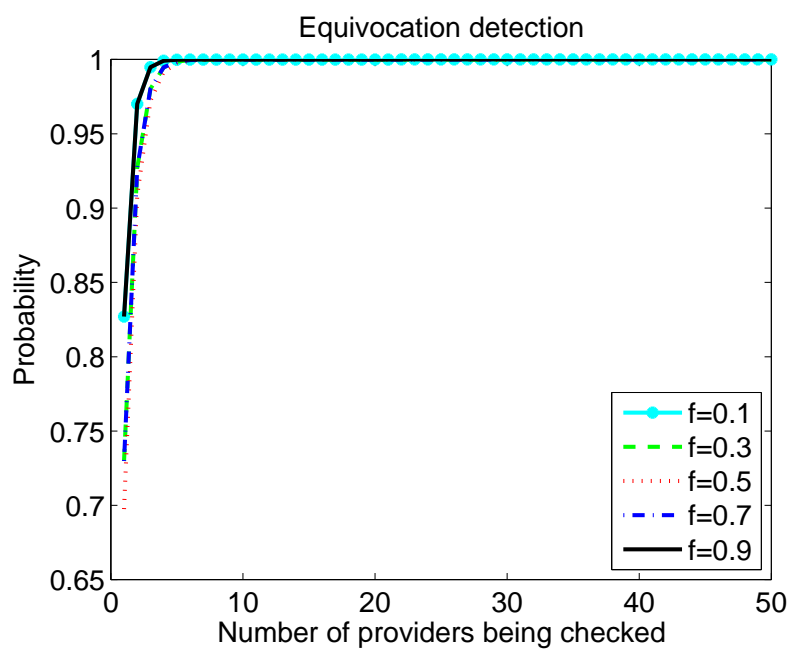

(b) Second scenario, $e=0.1$.

Figure 7: Equivocation detection.

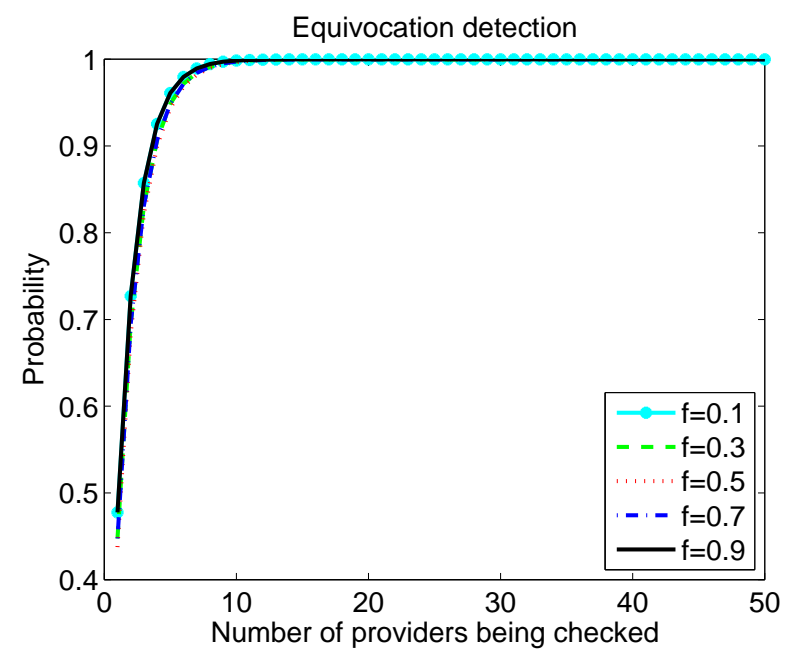

(a) $e=0.5$.

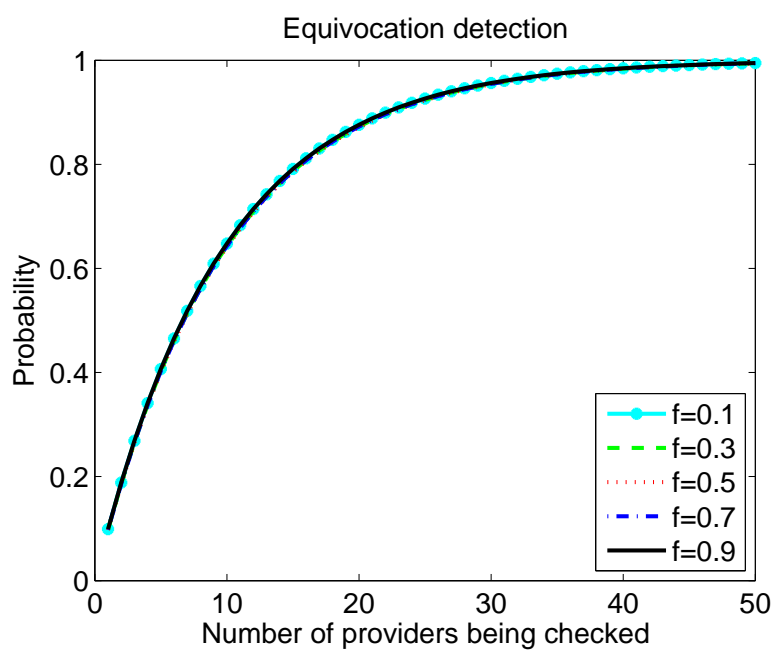

(b) $e=0.9$.

Figure 8: Equivocation detection, second scenario.

that increasing the portion of equivocating providers, decreases the probability of equivocation detection. However, when $e=0.9$, Alice and Bob can detect the equivocation with probability 0.9946 by challenging $k=50$ providers. 